\title{
Synthesis and Biological Evaluation of 3-Amino-4-aryl-piperidine Derivatives as BACE 1 Inhibitors
}

\author{
Hee-Jong Lim," Myung Hee Jung, Ihl Young Choi Lee, and Woo Kyu Park \\ Bio-Organic Science Division, Korea Research Institute of Chemical Technologv, P.O. Box 107, \\ Yustung-Gu, Daejeon 305-600, Korea. "E-mail: heejong@krict.re.kr \\ Received June 7, 2006
}

\begin{abstract}
$\mathrm{B} \Lambda \mathrm{CE} 1$ ( $\beta$-secretase), a membrane bound aspartic protease, is a key enzyme in the process of amyloid precursor protein $(\Lambda \mathrm{PP})$ into $\Lambda \beta$ peptide which is considered to play a causative role in $\Lambda$ lzheimer's Disease $(\Lambda D)$. Here, we reported the synthesis and inhibitory activity of optically active 3-amino-4-aryl-piperidines.
\end{abstract}

Key Words : Alzheimer's disease (AD), BACE I $(\beta$-secretase), Non-peptidomimetic inhibitors, Piperidine derivatives

\section{Introduction}

Alzheimer's disease $(\mathrm{AD})$ is a progressive neurodegenerative disease and most common form of dementia affecting about 20 millions worldwide, which has clinical symptoms of memory loss, impaiment of daily living activity, and ultimately leads to death. ${ }^{1} \mathrm{AD}$ is characterized pathologically by deposition of senile plaques and neurofibrillary tangles found in the brain of post-mortem $\mathrm{AD}$ patients. ${ }^{2}$ Amyloid $\beta$ peptide $(A \beta)$, a major component of senile plaques, is derived from amyloid precursor protein (APP) by sequential endoproteolysis by $\beta$-and $\gamma$-secretase. ${ }^{3}$

Numerous evidences suggest that an excessive level of $A \beta$ as soluble oligomers and filamentous fibrils initiate neuronal loss and play a causative role in the disease process. ${ }^{4}$ Consequently, inhibition of $\mathrm{A} \beta$ formation would prevent and suppress the development of AD. BACE 1 ( $\beta$-secretase), "a membrane bound aspartic protease, is a key enzyme responsible for APP processing into $A \beta$ peptide and thus considered a promising therapeutic target for the treatment of $\mathrm{AD} .^{6}$

Although numerous peptidomimetic BACE 1 inhibitors, derived from statine, hydroxyethylene, hydroxyethylamine, hydroxymethylcarbonyl, and aminoethylamine as a transition state mimic core motif frequently found in other aspartic protease inhibitors, are highly active in enzymatic assay, ${ }^{7}$ they would not be potential drug leads due to their poor cell permeability and bioavailability. Recently, endeavors to develop nonpeptide cell permeable BACE 1 inhibitors have been extensively studied, and various scaffolds such as isophthalamide, "diphenylurea, piperazine," and piperidine were reported to possess BACE 1 inhibitory activity.

4-Aryl-3-alkoxypiperidines have been reported to bind to carboxyl group of aspartic residue in the active site of human renin in a similar binding mode to the binding of peptidomimetic inhibitors derived from statin and aminostatin and shown very potent activity against renin, ${ }^{12}$ but they have not been fully studied in the development of BACE 1 inhibitors. " We anticipated new highly active nonpeptidomimetic BACE 1 inhibitors could be developed by modification of piperidine scaffold with appropriate substi- tuents. In this study, we present the synthesis and BACE 1 inhibitory activity of optically pure (3R)-amino-(4R)-arylpiperidine derivatives.

\section{Results and Discussion}

Chemistry. 3-Amino-4-arylpiperidines 6, key precursors for construction of various substituted piperidines, were prepared from $\mathrm{N}$-Boc-(L)-serine by modification of known literature procedure as shown in Scheme $1 .^{13}$

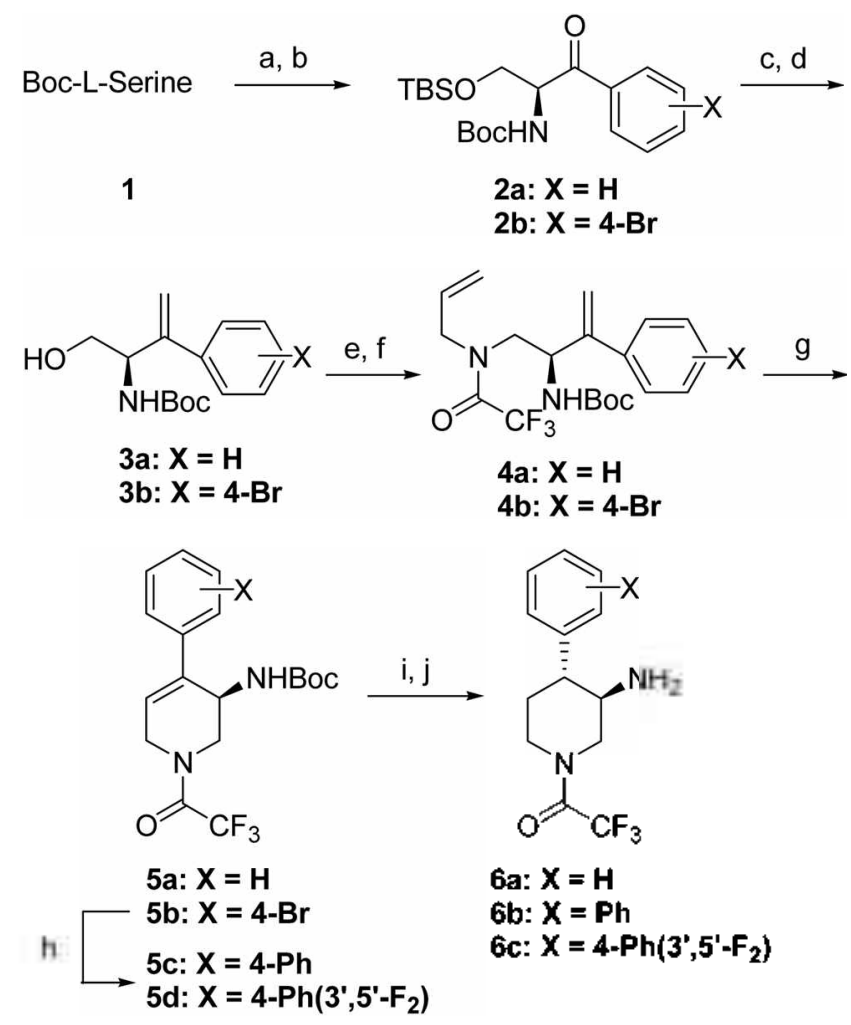

Scheme 1. Reagents; (a) TBS-Cl, Imidazolc. DMF; (b) (i) CDI, $\mathrm{THF}$, ii) $\wedge \mathrm{rMgBr}$, CuI; (c) MePPh;Br, $t$-BuOK, THF; (d) TBAF, THF; (e) Ms-Cl, TEA, $\mathrm{CH}_{2} \mathrm{Cl}_{2}$; (f) (i) Allylamine, (ii) Trifioroactic anhydride; (g) 2nd generation Grubbs Cat., $\mathrm{CH}_{2} \mathrm{Cl}_{2} \div(\mathrm{h}) \mathrm{ArB}(\mathrm{OH})_{2}$, $\mathrm{Pd}\left(\mathrm{PPh}_{3}\right)_{4}, \mathrm{Na}_{2} \mathrm{CO}_{3}$, aq.DME; (i) $\mathrm{H}_{2}, \mathrm{Pd}-\mathrm{C}, \mathrm{McOH}$; (i) $\mathrm{TF} \Lambda, \mathrm{CH}_{2} \mathrm{Cl}_{2}$. 


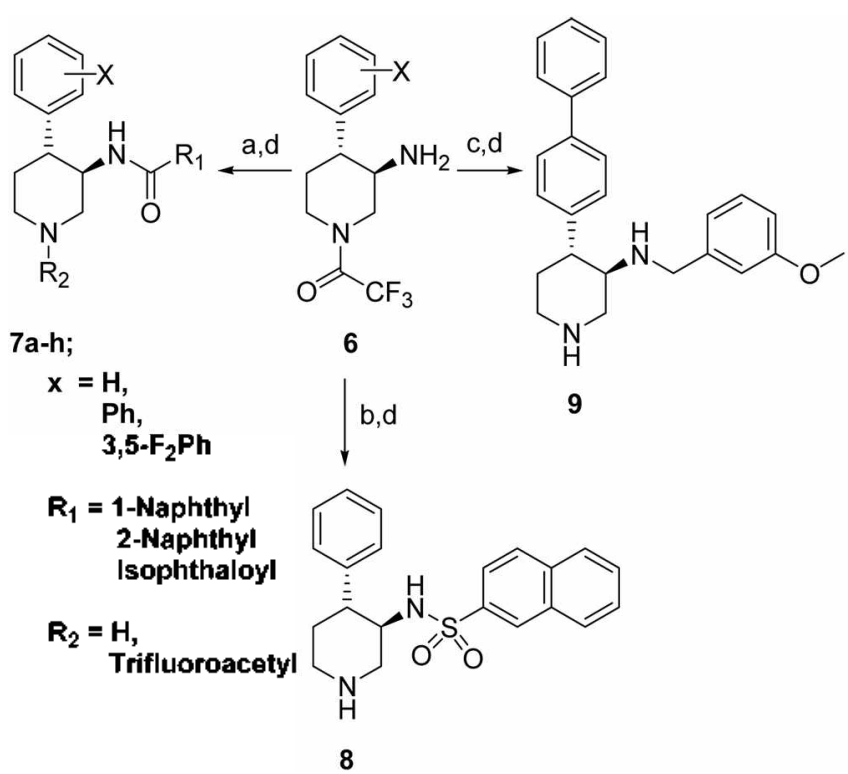

Scheme 2. Reagents; (a) ArCOCl, pyridine; (b) 2-Naphthalenesulfonyl chloride, pyridine; (c) 3-Methoxybenzylbromide, $\mathrm{K}_{2} \mathrm{CO}_{3}$, $\mathrm{DMF} ;$ (d) $\mathrm{K}_{2} \mathrm{CO}_{3}, \mathrm{MeOH}$.

Starting from Boc-L-serine, 2-aryl-3-amino-4-hydroxy-1butene 3, known precursors for 3-amino-4-aryl-piperidine ring 6, were readily prepared in four steps via protection of hydroxyl group to TBS ether, addition of aryl anion to activated acid, ${ }^{14}$ Wittig olefination of ketone 2 , and deprotection of TBS ether. It is noteworthy that this procedure has an advantage over the reported method for the preparation of compound 3 in overall 8 steps. ${ }^{13}$ With an improved synthesis of alcohol 3 in hand, the remaining steps to $(3 \mathrm{R})$-amino-( $4 \mathrm{R})$-aryl-piperidine 6 were undertaken as known procedure: transformation of hydroxyl group to the corresponding mesylate, substitution with allylamine, and followed by protection with trifluoroacetic anhydride to afford diene 4 . Ring closing metathesis of diene $\mathbf{4}$ with $2^{\text {nd }}$ generation Grubbs catalyst gave 3-t-Boc-amino-4-aryl1,2,3,6-tetrahydropyridine 5. 4-Bromphenyl compound $\mathbf{5 b}$ was converted to corresponding 4-biaryl compound $\mathbf{5 e}$ and 5d by a typical Suzuki coupling reaction with phenylboronic acid and 3,5-difluorophenylboronic acid, respectively. Finally, reduction of double bond and deprotection of Boc group gave desired 3-amino-4-aryl-piperdine 6.

Reaction of 3-amino-4-arylpiperidines 6 with various acid chloride, sulfonyl chloride, and 3-methoxybenzyl bromide and then treatment with $\mathrm{K}_{2} \mathrm{CO}_{3}$ in $\mathrm{MeOH}$ to remove trifluoroacetyl group gave amide 7a-h, sulfonamide 8 , and benzylamine 9 , respectively (Scheme 2 ).

\section{Results and Discussion}

The enzymatic inhibitory assay was carried out at the concentration of $100,10,2 \mu \mathrm{M}$ of a compound by a fluorescence resonance energy transfer (FRET) assay, using a purified baculovirus-expressed BACE I and a specific substrate (Rh-EVNLDAEFK-Quencher). The assay data were summarized in Table 1.

Initially we prepared 4-phenyl piperidine derivatives with amide- (7a-7d), sulfonamide- (8) substituents on 3-amino group. These 4-phenyl piperidines showed very little activity. Next, we decided to introduce a bulky substituent at 4position and prepared 4-biaryl substituted piperidines, which found in piperazine based BACE 1 inhibitors. ${ }^{10 t}$ 4-Biaryl piperidines $(\mathbf{7 e}, \mathbf{7 f}$, and $\mathbf{7 h}$ ) showed high inhibitory activities with $\mathrm{IC}_{50}$ of 2.8-9.8 $\mu \mathrm{M}$. In 4-biaryl pipperidine series, 3-(2naphtholyamino) piperidine ( $7 \mathrm{f}$ and $7 \mathrm{~h}$ ) showed $\mathrm{ca} .4$ fold higher activity than that of 4-phenyl-3-(4-biphenylcarbonyloxy)piperidine 10, which was previously identified as BACE 1 inhibitor with $\mathrm{IC}_{50}$ of $11 \mu \mathrm{M}$." Effect of substituent

Table 1. BACE 1 inhibitory activity of 3-amino-4-aryl-piperdines (7-9) and 10

\begin{tabular}{|c|c|c|c|c|c|}
\hline \multirow{2}{*}{ Compound } & \multirow{2}{*}{$\mathrm{X}$} & \multirow{2}{*}{$\mathrm{R}_{\mathbf{I}}$} & \multirow{2}{*}{$\mathrm{R}_{2}$} & \multicolumn{2}{|c|}{ Enzyme Assay } \\
\hline & & & & $\%$ inhibition (at $100 \mu \mathrm{M}$ ) & $\mathrm{IC}_{\leftarrow i j}$ \\
\hline $7 a$ & $\mathrm{H}$ & 4-Biphenyl & $\mathrm{H}$ & $26 \%$ & - \\
\hline $7 \mathbf{b}$ & $\mathrm{H}$ & 3-(Dipropylcarbamoyl)phenyl & $\mathrm{H}$ & $12 \%$ & - \\
\hline $7 \mathrm{c}$ & $\mathrm{H}$ & 3-(Dipropylcarbamoyl)phenyl & $\mathrm{CF}_{3} \mathrm{CO}^{-}$ & $17 \%$ & - \\
\hline $7 d$ & $\mathrm{H}$ & 3-(Diisopropylcarbamoyl)phenyl & $\mathrm{H}$ & $15 \%$ & - \\
\hline $7 e$ & 4-Ph & 1-Naphthyl & $\mathrm{H}$ & $50 \%$ & 9.83 \\
\hline $7 f$ & 4-Ph & 2-Naphthyl & $\mathrm{H}$ & $77 \%$ & 2.81 \\
\hline $7 \mathrm{~g}$ & 4-Ph & N,N-Dipropyl-isophthaloyl & $\mathrm{H}$ & $39 \%$ & - \\
\hline $7 \mathbf{h}$ & 4-Ph $\left(3,5-F_{2}\right)$ & 2-Naphthyl & $\mathrm{H}$ & $77 \%$ & 2.87 \\
\hline 8 & & & & $44 \%$ & - \\
\hline 9 & & & & $8.0 \%$ & - \\
\hline & & (10) & & & 11.0 \\
\hline
\end{tabular}

a. \% inhibition at $10 \mu \mathrm{M}$ 
in 3-amino group on inhibitory activity was also studied. 2Naphthoyl subsitutent (7f and $7 \mathbf{h}$ ) showed higher activity than 1-naphthoyl substituent (7e), and isophthaloyl $(7 \mathrm{~g})$ and benzyl group (9) showed poor activities.

In conclusion, we prepared 4-phenyl- and 4-biaryl-3amidopiperidine derivatives which showed better BACE 1 activities than originally reported compound 10. Further modification for improving activity and study of structure and activity relationship (SAR) are remained for future work.

\section{Experimental Section}

Melting points were determined on a capillary melting point apparatus and were uncorrected. 'H NMR spectra were recorded with Varian Gemini 200 spectrometer. Reagents used were reagent grade and purified, if necessary. Solvents were dried by conventional methods. In enzymatic assay, fluorescence intensity was read at $545 \mathrm{~nm}$ excitation and 585 nm emission with FlexStation fluorometric imaging reader.

Enzymatic assay. The inhibitory activity was determined by a fluorescence resonance energy transfer (FRET) assay in $50 \mathrm{mM}$ sodium acetate assay buffer at $\mathrm{pH} 4.5$, using a purified baculovirus-expressed BACE $1(1 \mathrm{U} / \mathrm{mL}$ in tris buffer) and a specific substrate (Rh-EVNLDAEFK-Quencher; $750 \mathrm{nM}$ in assay buffer). This specific substrate based on the Swedish mutation of the amyloid precursor protein (APP) became highly fluorescent upon cleavage by BACE 1 . For each compound, three different concentration $(100,10$, and $2 \mu \mathrm{M}$, respectively) were prepared by dissolving it in DMSO (up to $30 \%$ in total volume) and assay buffer.

After a solution of compound $(10 \mu \mathrm{L})$ and specific substrate $(10 \mu \mathrm{L})$ were gently mixed, BACE $1(10 \mu \mathrm{L})$ was introduced and incubated at room temperature for $1 \mathrm{~h}$. BACE 1 stop solution ( $2.5 \mathrm{M}$ sodium acetate buffer; $10 \mu \mathrm{L}$ ) was added to the reaction mixture to stop the reaction. Fluorescence intensity was measured to determine inhibitory activity of a compound tested. Each inhibitory assay was carried out repeatedly for three times.

O-tert-Butyldimethylsilyl- $N$-boc-L-serine. To a solution of N-Boc-L-serine (10.06 g. $49.03 \mathrm{mmol}$ ) in DMF ( $100 \mathrm{~mL}$ ) was added TBS-Cl $(9.46 \mathrm{~g}, 62.76 \mathrm{mmol})$ and imidazole $(10.01 \mathrm{~g}, 147.1 \mathrm{mmol})$ at $0^{\circ} \mathrm{C}$. After stirred for $10 \mathrm{~h}$ at $\mathrm{rt}$, the reaction mixture was diluted with water $(200 \mathrm{~mL})$ and extracted with ether $(2 \times 250 \mathrm{~mL})$. The combined organic layer was washed with water $(100 \mathrm{~mL})$, dried $\left(\mathrm{MgSO}_{4}\right)$, and concentrated. The remaining residue was purified by flash column chromatography (EtOAc: $\mathrm{Hex}=1: 4$ ) to give 13.3 $\mathrm{g}(85 \%)$ of O-t-butyldimethylsilyl-N-Boc-L-serine as a colorless oil: 'H NMR $\left(\mathrm{CDCl}_{3}\right): \delta 0.06(\mathrm{~s}, 6 \mathrm{H}), 0.88(\mathrm{~s}, 9 \mathrm{H})$, $1.46(\mathrm{~s}, 9 \mathrm{H}), 3.84(\mathrm{dd}, J=10.2,3.8 \mathrm{~Hz}, 1 \mathrm{H}), 4.12(\mathrm{~m}, 1 \mathrm{H})$, $4.35(\mathrm{~m}, 1 \mathrm{H}), 5.34(\mathrm{~d}, J=7.2 \mathrm{~Hz}, 1 \mathrm{H}), 8.40(\mathrm{bs}, 1 \mathrm{H})$.

Phenyl ketone (2a). A solution of $\mathrm{O}-i$-butyldimethylsilyl$\mathrm{N}$-Boc-L-serine $(7.02 \mathrm{~g}, 22.0 \mathrm{mmol})$ and 1,1 '-carbonyldiimidazole $(3.57 \mathrm{~g}, 22.0 \mathrm{mmol})$ in dry THF $(50 \mathrm{~mL})$ was stirred for $1 \mathrm{~h}$ at $\mathrm{rt}$, and then was added dropwise to a solution of phenylmagnesium bromide (3 M in hexane; 22
$\mathrm{mL}, 66 \mathrm{mmol})$ and CuI $(0.38 \mathrm{mg}, 1.98 \mathrm{mmol})$ in THF (50 $\mathrm{mL}$ ) at $-78^{\circ} \mathrm{C}$. After stirred at $-78^{\circ} \mathrm{C}$ for $1 \mathrm{~h}$, the reaction mixture was quenched with $1 \mathrm{~N} \mathrm{HCl}(50 \mathrm{~mL})$ and extracted with $\operatorname{EtOAc}(3 \times 100 \mathrm{~mL})$. The combined organic layer was washed with water $(2 \times 100 \mathrm{~mL})$, dried $\left(\mathrm{MgSO}_{4}\right)$, and concentrated. The remaining residue was purified by flash column chromatography (EtOAc: $\mathrm{Hex}=1: 8$ ) to give 6.58 $\mathrm{g}(78 \%)$ of $2 \mathrm{a}$ as a coloreless oil: 'H NMR $\left(\mathrm{CDCl}_{3}\right): \delta-0.13$ $(\mathrm{s}, 3 \mathrm{H}),-0.11(\mathrm{~s}, 3 \mathrm{H}), 0.76(\mathrm{~s}, 9 \mathrm{H}), 1.46(\mathrm{~s}, 9 \mathrm{H}), 3.86-4.99$ $(\mathrm{m}, 2 \mathrm{H}), 5.25-5.31(\mathrm{~m}, 1 \mathrm{H}), 5.68(\mathrm{~d}, J=7.6 \mathrm{~Hz}, 1 \mathrm{H}), 7.42-$ $7.63(\mathrm{~m}, 3 \mathrm{H}), 7.91-7.96(\mathrm{~m}, 2 \mathrm{H})$.

4-Bromophenyl ketone (2b). Compound $2 \mathrm{~b}$ was prepared with 4-bromophenyllithium ${ }^{15}$ as shown for $2 \mathrm{a}$ in $85 \%$ yield as a white solid: $\mathrm{mp} 46-47^{\circ} \mathrm{C}$; ' $\mathrm{H}$ NMR $\left(\mathrm{CDCl}_{3}\right): \delta$ $-0.12(\mathrm{~s}, 3 \mathrm{H}),-0.101(\mathrm{~s}, 3 \mathrm{H}), 0.76(\mathrm{~s}, 9 \mathrm{H}), 1.46(\mathrm{~s}, 9 \mathrm{H})$, $3.86-4.99(\mathrm{~m}, 2 \mathrm{H}), 5.25-5.31(\mathrm{~m}, 1 \mathrm{H}), 5.64(\mathrm{~d}, J=7.6 \mathrm{~Hz}$, $1 \mathrm{H}), 7.29(\mathrm{~d}, J=9.0 \mathrm{~Hz}, 2 \mathrm{H}), 7.48(\mathrm{~d}, J=7.6 \mathrm{~Hz}, 2 \mathrm{H}$ ).

(R)-tert-Butyl 1-hydroxy-3-phenylbut-3-en-2-ylearbamate (3a). To a stirred suspension of methyltriphenylphosphonium bromide $(4.5 \mathrm{~g}, 12.6 \mathrm{mmol})$ in dry THF ( 30 $\mathrm{mL}$ ) was added $\mathrm{n}$-BuLi $(1.6 \mathrm{M}$ in hexane; $8.4 \mathrm{~mL}, 13.4$ $\mathrm{mmol}$ ) at $0{ }^{\circ} \mathrm{C}$. The reaction mixture was warmed to $\mathrm{rt}$, stirred for $1 \mathrm{~h}$, and then cooled to $-78^{\circ} \mathrm{C}$. A solution of ketone $2 \mathrm{a}(1.5 \mathrm{~g}, 3.95 \mathrm{mmol})$ in $\mathrm{THF}(10 \mathrm{~mL})$ was added dropwise to above solution, and the resulting mixture was slowly warmed to $\mathrm{rt}$. After stirred for $1 \mathrm{~h}$, the reaction mixture was poured into cold $1 \mathrm{~N} \mathrm{HCl}(100 \mathrm{~mL})$ and extracted with ether $(50 \mathrm{~mL})$. The organic layer was washed with water $(100 \mathrm{~mL})$, dried $\left(\mathrm{MgSO}_{4}\right)$, and concentrated. The remaining residue was purified by flash column chromatography (EtOAc: Hex $=1: 20)$ to give $0.93 \mathrm{~g}(62 \%)$ of the desired compound: ${ }^{1} \mathrm{H}$ NMR $\left(\mathrm{CDCl}_{3}\right): \delta-0.06(\mathrm{~s}, 3 \mathrm{H})$, $-0.04(\mathrm{~s}, 3 \mathrm{H}), 0.86(\mathrm{~s}, 9 \mathrm{H}), 1.46(\mathrm{~s}, 9 \mathrm{H}), 3.50(\mathrm{dd}, J=9.8$, $3.6 \mathrm{~Hz}, 1 \mathrm{H}), 3.68(\mathrm{dd}, J=10.2,4.0 \mathrm{~Hz}, 1 \mathrm{H}), 4.67-4.73(\mathrm{~m}$, $1 \mathrm{H}), 5.10-5.12(\mathrm{~m}, 1 \mathrm{H}), 5.22(\mathrm{~s}, 1 \mathrm{H}), 5.30(\mathrm{~s}, 1 \mathrm{H}), 7.28-7.40$ (m, 5H).

Tetra- $n$-butylammonium fluoride $(1 \mathrm{M}$ in THF; $1.71 \mathrm{~mL}$, $1.71 \mathrm{mmol})$ was added to a solution of silyl ether $(0.59 \mathrm{~g}$, $1.56 \mathrm{mmol})$ in $\mathrm{CH}_{2} \mathrm{Cl}_{2}(10 \mathrm{~mL})$ at $0^{\circ} \mathrm{C}$. The reaction mixture was warned to $\mathrm{rt}$ and stirred for $1 \mathrm{~h}$. The reaction mixture was washed with water $(10 \mathrm{~mL})$, dried $\left(\mathrm{MgSO}_{4}\right)$, and concentrated. The remaining residue was purified by flash column chromatography (EtOAc : Hex $=1: 20$ ) to give $0.383 \mathrm{~g}(92 \%)$ of $3 \mathrm{an}$ ' 'H NMR $\left(\mathrm{CDCl}_{3}\right): \delta 1.47(\mathrm{~s}, 9 \mathrm{H}), 2.00$ (bs, $1 \mathrm{H}), 3.60-3.76(\mathrm{~m}, 2 \mathrm{H}), 4.75(\mathrm{~m}, 1 \mathrm{H}), 5.15(\mathrm{~m}, 2 \mathrm{H})$, $5.25(\mathrm{~s}, 1 \mathrm{H}), 5.39(\mathrm{~s}, 1 \mathrm{H}), 7.31-7.38(\mathrm{~m}, 5 \mathrm{H})$.

(R)-tert-Butyl 3-(4-bromophenyl)-1-hydroxybut-3-en2-ylcarbamate (3b). Compound $3 \mathrm{~b}$ was prepared from $\mathbf{2 b}$ as shown for 3a. White solid: mp $158-160^{\circ} \mathrm{C}$; ${ }^{1} \mathrm{H}$ NMR $\left(\mathrm{CDCl}_{3}\right): \delta 1.47(\mathrm{~s}, 9 \mathrm{H}), 1.68(\mathrm{bs}, 1 \mathrm{H}), 3.54-3.74(\mathrm{~m}, 2 \mathrm{H})$, $4.70(\mathrm{~m}, 1 \mathrm{H}), 5.15(\mathrm{~m}, 2 \mathrm{H}), 5.28(\mathrm{~s}, 1 \mathrm{H}), 5.39(\mathrm{~s}, 1 \mathrm{H}), 7.28$ $(\mathrm{d}, J=7.6 \mathrm{~Hz}, 2 \mathrm{H}), 7.47(\mathrm{~d}, J=8.6 \mathrm{~Hz}, 2 \mathrm{H})$.

(R)-tert-Butyl 1-( $N$-allyl-2,2,2-trifluoroacetamido)-3phenylbut-3-en-2-ylcarbamate 4a. To a solution of alcohol 3a $(1.15 \mathrm{~g}, 4.37 \mathrm{mmol})$ and triethylamine $(1.3 \mathrm{~mL}, 9.32$ mmol $)$ in $\mathrm{CH}_{2} \mathrm{Cl}_{2}(20 \mathrm{~mL})$ was added dropwise $\mathrm{MsCl}(0.41$ $\mathrm{mL}, 5.3 \mathrm{mmol}$ ) at $0^{\circ} \mathrm{C}$. The reaction mixture was warmed to 
rt, stirred for $1 \mathrm{~h}$, and washed with cold $1 \mathrm{~N} \mathrm{HCl}(20 \mathrm{~mL})$. The organic layer was dried $\left(\mathrm{MgSO}_{4}\right)$ and concentrated to give corresponding mesylate. Without purification, mesylate was dissolved in allylamine $(15 \mathrm{~mL})$ and heated to reflux ovemight under $\mathrm{N}_{2}$ atmosphere. The reaction mixture was concentrated and dissolved in $\mathrm{CH}_{2} \mathrm{Cl}_{2}(20 \mathrm{~mL})$ and treated sequentially with triethylamine $(1.3 \mathrm{~mL}, 9.32 \mathrm{mmol})$ and trifluoroacetic anhydride $(0.93 \mathrm{~mL}, 6.58 \mathrm{mmol})$ at $0^{\circ} \mathrm{C}$ and slowly warmed to $\mathrm{rt}$. After stirred for $2 \mathrm{~h}$, the reaction mixture was washed with cold $1 \mathrm{~N} \mathrm{HCl}(20 \mathrm{~mL})$ and water $(20 \mathrm{~mL})$. The organic layer was dried $\left(\mathrm{MgSO}_{4}\right)$, and concentrated. The remaining residue was purified by flash column chromatography (EtOAc: $\mathrm{Hex}=1: 6$ ) to give 1.59 $\mathrm{g}(91 \%)$ of $4 \mathrm{a}$ as a colorless oil: ${ }^{1} \mathrm{H} N \mathrm{NR}\left(\mathrm{CDCl}_{3}\right): \delta 1.43(\mathrm{~s}$, $9 \mathrm{H}), 3.16(\mathrm{dd}, J=13.8,3.4 \mathrm{~Hz}, 1 \mathrm{H}), 3.77-4.02(\mathrm{~m}, 3 \mathrm{H})$, 4.99-5.25(m, $4 \mathrm{H}), 5.30(\mathrm{~s}, 1 \mathrm{H}), 5.38(\mathrm{~s}, 1 \mathrm{H}), 5.58-5.66(\mathrm{~m}$, $1 \mathrm{H}), 7.29-7.44(\mathrm{~m}, 5 \mathrm{H})$.

(R)-tert-Butyl 1-( $N$-allyl-2,2,2-trifluoroacetamido)-3(4-bromophenyl)but-3-en-2-ylcarbamate $4 \mathrm{~b}$. Compound $4 b$ was prepared from $3 b$ as shown for $4 a$. White solid: $m p$ $137-139^{\circ} \mathrm{C} ;{ }^{1} \mathrm{H}$ NMR $\left(\mathrm{CDCl}_{3}\right): \delta 1.43(\mathrm{~s}, 9 \mathrm{H}), 3.16(\mathrm{dd}, J=$ $13.8,3.4 \mathrm{~Hz}, 1 \mathrm{H}), 3.77-4.02(\mathrm{~m}, 3 \mathrm{H}), 4.90-5.38(\mathrm{~m}, 4 \mathrm{H})$, $5.32(\mathrm{~s}, 1 \mathrm{H}), 5.38(\mathrm{~s}, 1 \mathrm{H}), 5.58-5.66(\mathrm{~m}, 1 \mathrm{H}), 7.29(\mathrm{~d}, J=9.0$ $\mathrm{Hz}, 2 \mathrm{H}), 7.48(\mathrm{~d}, J=7.6 \mathrm{~Hz}, 2 \mathrm{H})$.

(R)-tert-Butyl 4-phenyl-1-(2,2,2-trifluoroacetyl)-1,2,3, 6-tetrahydropyridin-3-ylcarbamate 5a. To a solution of diene $4 \mathrm{a}(0.83 \mathrm{~g}, 2.09 \mathrm{mmol})$ was added $2^{\text {nd }}$ generation Grubbs catalyst $(89 \mathrm{mg}, 0.104 \mathrm{mmol})$ and heated to reflux ovemight under argon atmosphere. The reaction mixture was concentrated and purified by flash column chromatography (EtOAc: $\mathrm{Hex}=1: 4)$ to give $0.68 \mathrm{~g}(87 \%)$ of $5 \mathrm{a}$ as a colorless oil: 'H NMR $\left(\mathrm{CDCl}_{3}\right): \delta 1.41(\mathrm{~m}, 9 \mathrm{H}), 3.45(\mathrm{dd}, J$ $=14.0,2.2 \mathrm{~Hz}, 1 \mathrm{H}), 3.80-3.91(\mathrm{~m}, 1 \mathrm{H}), 4.32-4.39(\mathrm{~m}, 1 \mathrm{H})$, 4.59-4.63 (m, 1H), 4.78-4.88 (m, 3H), 6.25-6.28 (m, 1H), 7.26-7.41 (m, 5H).

(R)-teri-Butyl 4-(4-bromophenyl)-1-(2,2,2-trifluoroacetyl)-1,2,3,6-tetrahydropyridin-3-ylearbamate $5 \mathrm{~b}$. Compound 5b was prepared from $4 b$ as shown for $5 \mathrm{a}$. White solid: $\mathrm{mp}$ $123-125^{\circ} \mathrm{C}$; 'H NMR $\left(\mathrm{CDCl}_{3}\right): \delta 1.41(\mathrm{~m}, 9 \mathrm{H}), 3.45(\mathrm{dd}, J=$ $13.8,2.0 \mathrm{~Hz}, 1 \mathrm{H}), 3.78-3.89(\mathrm{~m}, 1 \mathrm{H}), 4.29-4.36(\mathrm{~m}, 1 \mathrm{H})$, $4.57-4.86(\mathrm{~m}, 3 \mathrm{H}), 6.25-6.28(\mathrm{~m}, 1 \mathrm{H}), 7.28(\mathrm{~d}, J=9.4 \mathrm{~Hz}$, $2 \mathrm{H}), 7.50$ (d, $J=8.4 \mathrm{~Hz}, 2 \mathrm{H})$.

(R)-teri-Butyl 4-(biphenyl-4-yl)-1-(2,2,2-trifluoroacetyl)1,2,3,6-tetrahydropyridin-3-ylcarbamate 5 c. To a solution of $5 \mathrm{~b}(1.0 \mathrm{~g}, 2.22 \mathrm{mmol})$, phenylboronic acid $(0.3 \mathrm{~g}$, $2.46 \mathrm{mmol})$, and CsF $(0.67 \mathrm{~g}, 4.41 \mathrm{mmol})$ in DME $(10 \mathrm{~mL})$ was added tetrakis (triphenylphosphine) palladium $(77 \mathrm{mg}$, $66 \mu \mathrm{mol})$. The reaction mixture was heated to reflux ovemight under argon atmosphere. After cooled to rt, the reaction mixture was diluted with EtOAc $(30 \mathrm{~mL})$, washed with water $(50 \mathrm{~mL})$, dried $\left(\mathrm{MgSO}_{4}\right)$, and concentrated. The remaining residue was purified by flash column chromatography (EtOAc : Hex $=1: 6$ ) to give $0.84 \mathrm{~g}(84 \%)$ of $\mathbf{5 b}$ as a colorless oil: 'H NMR $\left(\mathrm{CDCl}_{3}\right): \delta 1.42(\mathrm{~s}, 9 \mathrm{H}), 3.46(\mathrm{dd}, J=$ $13.8,2.0 \mathrm{~Hz}, 1 \mathrm{H}), 3.82-3.92(\mathrm{~m}, 1 \mathrm{H}), 4.33-4.41(\mathrm{~m}, 1 \mathrm{H})$, $4.64-4.90(\mathrm{~m}, 3 \mathrm{H}), 6.31-6.34(\mathrm{~m}, 1 \mathrm{H}), 7.32-7.63(\mathrm{~m}, 9 \mathrm{H})$.

(R)-tert-Butyl 4-(3',5'-difluorobiphenyl-4-yl)-1-(2,2,2- trifluoroacetyl)-1,2,3,6-tetrahydropyridin-3-ylcarbamate 5d. Compound 5d was prepared from 5b with 3,5-difluorophenylboronic acid as shown for $\mathbf{5 c} .{ }^{1} \mathrm{H}$ NMR $\left(\mathrm{CDCl}_{3}\right): \delta$ $1.41(\mathrm{~s}, 9 \mathrm{H}), 3.43-3.54(\mathrm{~m}, 1 \mathrm{H}), 3.80-3.89(\mathrm{~m}, 1 \mathrm{H}), 4.30-$ $4.39(\mathrm{~m}, 1 \mathrm{H}), 4.57-4.94(\mathrm{~m}, 3 \mathrm{H}), 6.29-6.30(\mathrm{~m}, 1 \mathrm{H}), 6.67-$ $6.76(\mathrm{~m}, 1 \mathrm{H}), 6.99-7.09(\mathrm{~m}, 2 \mathrm{H})$, 7.34-7.57 (m, 7H), 7.71$7.92(\mathrm{~m}, 4 \mathrm{H}), 8.21(\mathrm{~s}, 1 \mathrm{H})$.

1-((3R,4R)-3-Amino-4-phenylpiperidin-1-yl)-2,2,2-trifluoroethanone 6a. A solution of tetrahydropyridine 5a $(0.67 \mathrm{~g}, 1.82 \mathrm{mmol})$ and $5 \%$ Pd-C $(97 \mathrm{mg}, 0.91 \mathrm{mmol})$ in $\mathrm{MeOH}(20 \mathrm{~mL})$ was stirred under hydrogen balloon for $4 \mathrm{~h}$. The reaction mixture was filtered over celite pad and concentrated in vacuo. The remaining residue was purified by flash column chromatography (EtOAc : $\mathrm{Hex}=1: 8$ ) to give 3-tert-butoxycarbonyl-amino-piperidine. To a solution of 3-tert-butoxycarbonyl-amino-4-phenyl-piperidine in $\mathrm{CH}_{2} \mathrm{Cl}_{2}(10 \mathrm{~mL})$ was added TFA $(3 \mathrm{~mL})$ at $0^{\circ} \mathrm{C}$. The reaction mixture was slowly warmed to it and stirred for $1 \mathrm{~h}$. The reaction mixture was concentrated in vacuo, diluted with EtOAc $(20 \mathrm{~mL})$, washed with sat'd $\mathrm{NaHCO}_{3}(20 \mathrm{~mL})$, and water $(20 \mathrm{~mL})$. The organic layer was dried $\left(\mathrm{MgSO}_{4}\right)$, concentrated, and purified by flash column chromatography (EtOAc : Hex $=1: 1)$ to give $0.59 \mathrm{~g}(87 \%)$ of $6 \mathrm{a}:{ }^{1} \mathrm{H} \mathrm{NMR}$ $\left(\mathrm{CDCl}_{3}\right): \delta 1.09(\mathrm{bs}, 2 \mathrm{H}), 1.71-1.78(\mathrm{~m}, 1 \mathrm{H}), 2.20-2.38(\mathrm{~m}$, $1 \mathrm{H}), 2.80-3.51(\mathrm{~m}, 4 \mathrm{H}), 3.95-4.28(\mathrm{~m}, 1 \mathrm{H}), 4.59-4.85(\mathrm{~m}$, $1 \mathrm{H}), 7.19-7.40(\mathrm{~m}, 5 \mathrm{H})$.

1-((3R,4R)-3-Amino-4-(biphenyl-4-yl)piperidin-1-yl)2,2,2-trifluoroethanone $6 \mathrm{~b}$. Compound $6 \mathrm{~b}$ was prepared from $5 \mathrm{c}$ as shown for $6 \mathrm{a}$. ${ }^{1} \mathrm{H}$ NMR $\left(\mathrm{CDCl}_{3}\right): \delta 1.16-1.26(\mathrm{~m}$, $1 \mathrm{H}), 1.86-2.02(\mathrm{~m}, 1 \mathrm{H}), 2.55-2.92(\mathrm{~m}, 1 \mathrm{H}), 3.07-3.32(\mathrm{~m}$, $3 \mathrm{H}), 3.74(\mathrm{~s}, 1 \mathrm{H}), 4.18-4.25(\mathrm{~m}, 1 \mathrm{H}), 4.86-4.94(\mathrm{~m}, 1 \mathrm{H})$, 7.32-7.62 (m, 9H).

1-((3R,4R)-3-Amino-4-(3',5'-difluorobiphenyl-4-yl)piperidin-1-yl)-2,2,2-trifluoroethanone $6 c$. Compound $6 \mathrm{c}$ was prepared from $5 \mathrm{~d}$ as shown for $6 \mathrm{a} .{ }^{1} \mathrm{H} \mathrm{NMR}\left(\mathrm{CDCl}_{3}\right): \delta 1.19-$ $1.26(\mathrm{~m}, 2 \mathrm{H}), 1.81-1.95(\mathrm{~m}, 1 \mathrm{H}), 2.64-2.88(\mathrm{~m}, 1 \mathrm{H}), 3.08-$ $3.42(\mathrm{~m}, 3 \mathrm{H}), 3.77(\mathrm{~s}, 1 \mathrm{H}), 4.02-4.27(\mathrm{~m}, 1 \mathrm{H}), 4.85-4.92(\mathrm{~m}$, $1 \mathrm{H}), 6.73-6.81(\mathrm{~m}, 1 \mathrm{H}), 7.02-7.05(\mathrm{~m}, 2 \mathrm{H}), 7.35-7.39(\mathrm{~m}$, $2 \mathrm{H}), 7.52-7.56(\mathrm{~m}, 2 \mathrm{H})$.

General procedure for the synthesis of amide $7 \mathrm{a}-7 \mathrm{~h}$. To a solution of 3-aminopiperidine 6 and triethylamine in $\mathrm{CH}_{2} \mathrm{Cl}_{2}(10 \mathrm{~mL})$ was added acid chloride at $0{ }^{\circ} \mathrm{C}$. The reaction mixture was warmed to it and stirred overnight. The re action mixture was washed with $1 \mathrm{~N} \mathrm{HCl}(10 \mathrm{~mL})$, aq. $\mathrm{NaHCO}_{3}(10 \mathrm{~mL})$, and water $(10 \mathrm{~mL})$. The organic layer was dried $\left(\mathrm{MgSO}_{4}\right)$, concentrated, and purified by flash column chromatography (EtOAc : Hex $=1: 1$ ) to yield the desired amide.

The corresponding $\mathrm{N}$-trifluoroacetylpiperidine was treated with $\mathrm{K}_{2} \mathrm{CO}_{3}$ in $\mathrm{MeOH}(3 \mathrm{~mL})$ at it for $1-4 \mathrm{~h}$. The reaction mixture concentrated and purified by flash column chromatography (EtOAc: $\mathrm{Hex}=1: 1$ ) to give desired free piperidine 7.

$N$-(3R,4R)-4-Phenylpiperidin-3-yl)biphenyl-4-carboxamide 7a. ${ }^{1} \mathrm{H} \mathrm{NMR}\left(\mathrm{CDCl}_{3}\right): \delta 1.80-1.95(\mathrm{~m}, 1 \mathrm{H}), 2.0-2.15$ $(\mathrm{m}, 1 \mathrm{H}), 2.78-2.88(\mathrm{~m}, 1 \mathrm{H}), 3.00-3.10(\mathrm{~m}, 2 \mathrm{H}), 3.20-3.25$ $(\mathrm{m}, 2 \mathrm{H}), 4.57-4.62(\mathrm{~m}, 1 \mathrm{H}), 6.93(\mathrm{~d}, \mathrm{~J}=8.0 \mathrm{~Hz}, 1 \mathrm{H}), 7.20-$ 
$7.72(\mathrm{~m}, 14 \mathrm{H})$.

$N^{-1}$-((3R,4R)-4-Phenylpiperidin-3-yl)- $N^{3}, N^{3}$-dipropylisophthalamide $7 \mathrm{~b} .{ }^{1} \mathrm{H}$ NMR $\left(\mathrm{CDCl}_{3}\right): \delta 0.75(\mathrm{bt}, 3 \mathrm{H}), 0.98$ (bt, $3 \mathrm{H}), 1.40-1.45(\mathrm{~m}, 2 \mathrm{H}), 1.62-1.65(\mathrm{~m}, 2 \mathrm{H}), 1.81-1.87(\mathrm{~m}$, $1 \mathrm{H}), 2.09-2.16-2.28(\mathrm{~m}, 2 \mathrm{H}), 2.83(\mathrm{~m}, 1 \mathrm{H}), 2.97-3.42(\mathrm{~m}$, $9 \mathrm{H}), 4.62-4.65(\mathrm{~m}, 1 \mathrm{H}), 7.15-7.44(\mathrm{~m}, 9 \mathrm{H})$.

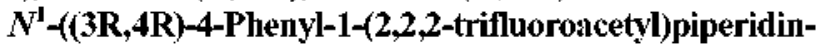
3-yl)- $N^{3}, N^{3}$-dipropylisophthalamide $7 \mathrm{c}$. 'H NMR $\left(\mathrm{CDCl}_{3}\right)$ : $\delta 0.75(\mathrm{bt}, 3 \mathrm{H}), 0.98(\mathrm{bt}, 3 \mathrm{H}), 1.40-1.80(\mathrm{~m}, 5 \mathrm{H}), 2.04-2.15$ $(\mathrm{m}, 2 \mathrm{H}), 3.14-3.45(\mathrm{~m}, 7 \mathrm{H}), 4.39-4.40(\mathrm{~m}, 2 \mathrm{H}), 6.44(\mathrm{bs}$, $1 \mathrm{H}), 7.15-7.44(\mathrm{~m}, 9 \mathrm{H})$.

$N^{1}, N^{1}-$ Diisopropyl- $N^{3}$-(3R,4R)-4-phenyl-1-(2,2,2-trifluoroacetyl)piperidin-3-yl)isophthalamide $7 \mathrm{~d} .{ }^{1} \mathrm{H} \mathrm{NMR}\left(\mathrm{CDCl}_{3}\right)$ : $\delta 0.75$ (bt, 3H), $0.98(\mathrm{bt}, 3 \mathrm{H}), 1.40-1.45(\mathrm{~m}, 2 \mathrm{H}), 1.62-1.65(\mathrm{~m}$, $2 \mathrm{H}), 1.81-1.87(\mathrm{~m}, 1 \mathrm{H}), 2.09-2.16-2.28(\mathrm{~m}, 2 \mathrm{H}), 2.83(\mathrm{~m}, 1 \mathrm{H})$, 2.97-3.42 (m, 9H), 4.62-4.65 (m, 1H), 7.15-7.44 (m, 9H).

$\mathrm{N}$-((3R,4R)-4-(Biphenyl-4-yl)piperidin-3-yl)-1-naphthamide 7e. ${ }^{1} \mathrm{H}$ NMR $\left(\mathrm{CDCl}_{3}\right): \delta 1.80-1.97(\mathrm{~m}, 2 \mathrm{H}), 2.65(\mathrm{~s}$, $2 \mathrm{H}), 2.79$ (dt, $J=11.4,2.8 \mathrm{~Hz}, 1 \mathrm{H}), 3.04-3.33(\mathrm{~m}, 3 \mathrm{H}), 4.85$ (d, $J=6.8 \mathrm{~Hz}, 1 \mathrm{H}), 6.90-6.95(\mathrm{~m}, 1 \mathrm{H}), 7.21-7.24(\mathrm{~m}, 1 \mathrm{H})$, 7.39- $7.62(\mathrm{~m}, 12 \mathrm{H}), 7.74-7.84(\mathrm{~m}, 2 \mathrm{H})$.

$N$-((3R,4R)-4-(Biphenyl-4-yl)piperidin-3-yl)-2-naphthamide 7f. ' $\mathrm{H}$ NMR $\left(\mathrm{CDCl}_{3}\right): \delta 1.86(-1.92(\mathrm{~m}, 1 \mathrm{H}), 2.06-$ $2.21(\mathrm{~m}, 1 \mathrm{H}), 2.35(\mathrm{~s}, 2 \mathrm{H}), 2.85(\mathrm{dt}, J=11.6,2.6 \mathrm{~Hz}, 1 \mathrm{H})$, $3.01-3.14(\mathrm{~m}, 2 \mathrm{H}), 3.25-3.30(\mathrm{~m}, 2 \mathrm{H}), 4.71(\mathrm{~d}, J=6.4 \mathrm{~Hz}$, $1 \mathrm{H}), 7.13-7.90(\mathrm{~m}, 15 \mathrm{H}), 8.16(\mathrm{~s}, 1 \mathrm{H})$.

$N^{1}$-((3R,4R)-4-(Biphenyl-4-yl)piperidin-3-yl)- $N^{3}, N^{3}$-dipropylisophthalamide $7 \mathrm{~g} .{ }^{1} \mathrm{H}$ NMR $\left(\mathrm{CDCl}_{3}\right): \delta 0.66(\mathrm{bt}$, $3 \mathrm{H}), 0.96(\mathrm{bt}, 3 \mathrm{H}), 1.43-1.47(\mathrm{~m}, 2 \mathrm{H}), 1.64-1.67(\mathrm{~m}, 2 \mathrm{H})$, $1.83-1.89(\mathrm{~m}, 1 \mathrm{H}), 2.09-2.16-2.28(\mathrm{~m}, 2 \mathrm{H}), 2.83(\mathrm{dt}, J=$ $11.8,2.8 \mathrm{~Hz}, 1 \mathrm{H}), 2.99-3.44(\mathrm{~m}, 9 \mathrm{H}), 4.62-4.65(\mathrm{~m}, 1 \mathrm{H})$, 7.03-7.07 (m, 1H), 7.26-7.68 (m, 12H).

$N^{1}$-((3R,4R)-4-(3',5'-Difluorobiphenyl-4-yl)piperidin3-yl)-2-naphthamide $7 \mathbf{h}$. 'H NMR ( $\left.\mathrm{CDCl}_{3}\right): \delta$ 1.85-1.91 (m, $1 \mathrm{H}), 2.10-2.31(\mathrm{~m}, 1 \mathrm{H}), 2.81-2.93(\mathrm{~m}, 1 \mathrm{H}), 3.05-3.12$ (m, $2 \mathrm{H}), 3.23-3.50(\mathrm{~m}, 2 \mathrm{H}), 4.74(\mathrm{~d}, J=9.4 \mathrm{~Hz}, 1 \mathrm{H}), 6.66-$ $6.76(\mathrm{~m}, 1 \mathrm{H}), 6.98-7.08(\mathrm{~m}, 2 \mathrm{H}), 7.34-7.57(\mathrm{~m}, 7 \mathrm{H})$.

$N$-((3R,4R)-4-Phenylpiperidin-3-yl)naphthalene-2-sulfonamide 8. To a solution of aminopiperidine $6 \mathrm{a}(40 \mathrm{mg}, 0.15$ mmol) and triethylamine ( $61 \mu \mathrm{L}, 0.43 \mathrm{mmol}$ ) was added in $\mathrm{CH}_{2} \mathrm{Cl}_{2}(10 \mathrm{~mL})$ was 2-naphthalenesulfonyl chloride (40) $\mathrm{mg}, 0.18 \mathrm{mmol}$ ). The reaction mixture was stirred for $4 \mathrm{~h}$ at rt, diluted with EtOAc, and washed with $1 \mathrm{~N} \mathrm{HCl}(10 \mathrm{~mL})$, sat'd $\mathrm{NaHCO}_{3}(10 \mathrm{~mL})$, and water $(10 \mathrm{~mL})$. The organic layer was dried $\left(\mathrm{MgSO}_{4}\right)$, concentrated, and purified by flash column chromatography (EtOAc: $\mathrm{Hex}=1: 3$ ) to give sulfonamide.

The corresponding sulfonamide was treated with $\mathrm{K}_{2} \mathrm{CO}_{3}$ in $\mathrm{MeOH}(3 \mathrm{~mL})$ at $\mathrm{rt}$ for $4 \mathrm{~h}$. The reaction mixture was concentrated and purified by flash column chromatography (EtOAc : Hex $=2: 1$ ) to give $38 \mathrm{mg}(69 \%)$ of desired sulfonamide 8. ${ }^{1} \mathrm{H}$ NMR ( $\left(\mathrm{CDCl}_{3}\right): \delta 1.6-2.1(\mathrm{~m}, 3 \mathrm{H}), 2.60-$ $2.90(\mathrm{~m}, 3 \mathrm{H}), 3.18-3.24(\mathrm{~m}, 2 \mathrm{H}), 3.55(\mathrm{~s}, 1 \mathrm{H}), 6.85-6.93(\mathrm{~m}$, $5 \mathrm{H}), 7.25-7.35(\mathrm{~m}, 2 \mathrm{H}), 7.56-7.61(\mathrm{~m}, 2 \mathrm{H}), 8.03(\mathrm{~s}, 1 \mathrm{H})$.

(3R,4R)-4-(Biphenyl-4-yl)- $N$-(3-methoxybenzyl)piperidin3-amino 9. To a solution of aminopiperidine $6 \mathrm{~b}(40 \mathrm{mg}$, $0.115 \mathrm{mmol}$ ) and $\mathrm{K}_{2} \mathrm{CO}_{3}(18 \mathrm{mg}, 0.13 \mathrm{mmol})$ was added $3-$ methoxybenzyl bromide ( $18 u \mathrm{~L}, 0.13 \mathrm{mmol}$ ). The reaction mixture was stirred overnight at $\mathrm{rt}$, diluted EtOAc, and washed with $1 \mathrm{~N} \mathrm{HCl}(10 \mathrm{~mL})$ and water $(10 \mathrm{~mL})$. The organic layer was dried $\left(\mathrm{MgSO}_{4}\right)$, concentrated, and purified by flash column chromatography (EtOAc: $\mathrm{Hex}=1: 1$ ) to give corresponding benzylamine.

The benzylamine was treated with $\mathrm{K}_{2} \mathrm{CO}_{3}$ in $\mathrm{MeOH}(3$ $\mathrm{mL}$ ) at $\mathrm{rt}$ for $4 \mathrm{~h}$. The reaction mixture concentrated and purified by flash column chromatography (EtOAc : Hex $=3$ :1) to give $35 \mathrm{mg}(83 \%)$ of desired benzylamine derivatives 9. ${ }^{1} \mathrm{H}$ NMR $\left(\mathrm{CDCl}_{3}\right): \delta 1.75-1.80(\mathrm{~m}, \mathrm{lH}), 2.02-2.04(\mathrm{~m}$, $1 \mathrm{H}), 2.64-2.98(\mathrm{~m}, 4 \mathrm{H}), 3.20(\mathrm{~m}, 2 \mathrm{H}), 3.72(\mathrm{~s}, 3 \mathrm{H}), 4.10-$ $4.17(\mathrm{~m}, 1 \mathrm{H}), 4.89(\mathrm{~s}, 2 \mathrm{H}), 5.79(\mathrm{~d}, J=9.4 \mathrm{~Hz}, 1 \mathrm{H}), 6.79(\mathrm{~m}$, $2 \mathrm{H}), 7.12-7.16(\mathrm{~m}, 1 \mathrm{H}), 7.25-7.58(\mathrm{~m}, 10 \mathrm{H})$.

\section{References}

I. Iqbal, K.; Sisodia, S. S.; Winblad, B. Alzheimer S Disease: Actiances in Etiolog; Pathogenesis and Therapetifics; John Wiley \& Son: England, $200 \mathrm{l}$.

2. (a) Selkoe, D. J. Natme 1999, 399, A23. (b) Roggo, A. Curr. Top. Med. Chem. 2002, 2, 359. (c) Vassar, R. Science 1999, 286, 375.

3. (a) Sinha, S.; Lieberburg, I. Proc. Natl. Acad. Sci. U.S.A. 1999, 96 , 11049. (b) Citron, M.; Diehl, T. S.; Gordon, G.; Biere, A. L.; Seubert, P.; Selkoe, D. J. Proc. Natl. Acad. Sci. U.S.A. 1996, 93, 13170 .

4. (a) Hardy, J.; Selkoe, D. J. Science 2002, 297, 353. (b) Hsiao, K.; Chapman, P.; Nilsen, S.; Eckman, C.; Harigaya, Y.; Younkin, S.; Yang, F.; Cole, G. Science 1996, 274, 99.

5. (a) Lin, X.; Koelsch, G; Wu, S.; Downs, D.; Dashti, A.; Tang, J. Proc. Natl. Acad. Sci. U.S.A. 2000, 97, 1456. (b) Hussain, I.; Powell, D.; Howlett. D. R.; Tew, D. G.; Meek, T. D.; Chapman, C.; Gloger, I. S.; Murphy, K. E.; Southan, C. D.; Ryan, D. M.; Smith, T. S.; Simmons, D. L.; Walsh, F. S.; Dingwall, C.; Christie, G. Mol. Cell Netrosci. 1999, 14,419. (c) Yan, R.; Bienkowski, M. J.; Shuck, M. E.; Miao, H.; Tory, M. C.; Pauley, A. M.; Brashier, J. R.; Stratman, N. C.; Mathews, W. R.; Buhl, A. E.; Carter, D. B.; Tomasselli, A. G.; Parodi, L. A.; Heinrikson, R. L.; Gurney, M. E. Nature 1999, 402, 533. (d). Sinha, S.; Anderson, J. P.; Barbour, R.; Basi, G. S.; Caccavello, R,; Davis, D.; Doan, M.; Dovey, H. F.; Frigon, N.; Hong, J.; Jacobson-Croak, K.; Jewetl, N.; Keim, P.; Knops, J.; Lieberburg, I.; Power, M.; Tan, H.; Tatsuno, G.; Tung, J.; Schenk, D.; Seubert, P.; Suomensaari, S. M.; Wang, S.; Walker, D.; John, V. Nature 1999, 402, 537. (e) Vassar, R.; Bennett, B. D.; Babu-Kahn, S.; Kahn, S.; Mendiaz, E. A.; Denis, P.; Teplow, D. B.; Ross, S.; Amarante, P.; Loeloff, R.; Luo, Y; Fisher, S.; Fuller, J.; Edenson, S.; Lile, J.; Jarosinski, M. A.; Biere, A. L.; Curran, E.; Burgess, T.; Louis, J. C.; Collins, F.; Treanor, J.; Rogers, G.; Citron, M. Science 1999, 286, 735.

6. (a) Wolf, M. S. Nature Rev. Drug Discovery 2002, 1,859 . (b) Citron, M. Nature Rev, Netwosci. 2004, 5, 677. (c) Gosh, A. K.; Hong, L.; Tang, J. Curr, Med. Chem. 2002, 9, 1135 . (d)Walter, J.; Haass, C. Ding Develop. Res. 2002, 56, 201, (e) Vassar, R. Adv. Drtig Del. Rev. 2002, 54, 1589.

7. (a) Gosh, A. K.; Shin, D. W.; Downs, D.; Koelsch, G; Lin, X.; Ermolieff, J.; Tnag, J. J. Am. Chen. Soc. 2000, 122, 3522. (b) John, V.; Beck, J. P.; Bienkowski, M. J.; Sinha, \$.; Heinrikson, R. L. J. Med. Chem, 2002, 46, 4625. (c) Hom, R. K.; Gailunas, A. F.; Mamo, S.; Fang, L. Y.; Tung, J. S.; Walker, D. E.; Davis, D.; Thorsett, E. D.; Jewett, N. E.; Moon, J. B.; John, V. J. Med. Chem. $2004,47,158$. (d) Chen, S.; Lamar, J.; Guo, D.; Kohn, T.; Yang. H.; McGee, J.; Timm, D.; Erickson, J.; Yip, Y.; May, P.; McCarthy, J. Bioorg. Med. Chem, Lett. 2004, 14, 245, (e) Hanessian, S.; Yun, H.; Hou, Y;; Yang, G; Bayrakdarian, M.; Therrien, E.; Moitessier, N.; Roggo, S.; Veenstra, S. et al. J. Med. 
Chem. 2005, 48, 5175. (f) Yang, W; Lu, W; Lu, Y; Zhong, M.; Sun, J.; Thomas, A. E.; Wilkinson, J. M.; Fucini, R. V.; Lam, M.; Randal, M.; Shi, X.-P.; Jacobs, J. W.; McDowell, R. S.; Gordon, E. M.; Ballinger, M. D. J. Med. Chen. 2006, 49, 839 .

8. Stachel, S. J.; Coburn, C. A.; Steele, T. G.; Jones, K. G.; Loutzenhiser, E. F.; Gregro, A. R.; Rajapakse, H. A.; Lai, M. T.; Crouthamel, M.-C.; Xu, M.; Tugusheva, K.; Lineberger, J. E.; Pietrak, B. L.; Espeseth, A. S.; Shi, X.-P; Chen-Dodson, E.; Holloway, M. K.; Munshi, S.; Simon, A. J.; Kuo, L.; Vacea, J. P. J. Med. Chem. 2004, 47,6447.

9. Huang, D.; Luthi, U.; Kolb, P.; Edler, K.; Cecchini, M.; Audetat, S.; Barberis, A.; Caflisch, A. J. Med. Chem. 2005, 48, 5108 .

10. (a) Park, H.; Lee, S. J. Am. Chem. Soc. 2003, 125, 16416. (b) Bhisetti, G. R.; Saunders, J. O.; Murcko, M. A.; Lepre, C. A.; Britt, S. D.; Come, J. H.; Deninger, D. D.; Wang, T. PCT Int. Appl. WO 02/088101, 2002. (c) Garino, C.; Pietrancosta, N.; Laras, Y.;
Moret, V.; Rolland, A.; Quelever, G.; Kraus, J.-L. Bioorg. Med. Chen. Letf. 2006, 16, 1995.

I1. John, V; Moon, J. B.; Pulley, S. R.; Rich, D. H.; Brown, D. L.; Jagodzinsak, B.; Jacobs, J. S. PCT Int. Appl. WO 03/04,3987, 2003.

12. (a) Bursarvich, M. G.; West, C. W.; Rich, D. H. Org. Lett, 2001, 3, 2317. (b) Oefner, C.; Binggeli, A.; Breu, V.; Bur, D.; Clozel, J. P.; D'arcy, A.; Dorn, A.; Fischli, W.; Gruninger, F.; Guller, R.; Hirth, G.; Marki, H.; Mathews, S.; Miller, M.; Ridley, R. G.; Stadler, H.; Viera, E.; Wilhelm, M.; Winkler, F.; Wostl, W. Chem. Biol. 1999, 6,127 .

13. Hu, X. E.; Kim, N. K.; Ledoussal, B. Org. Lett. 2002, 4, 4499.

14. Bonini, B. F.; Comes-Franchini, M.; Fochi, M.; Mazzanti, G.; Ricci, A.; Varchi, G. Synlett 1998, 1013.

15. Rogers, H. R,; Houk, J. J. Am. Chem. Soc. 1982, 104, 522. 